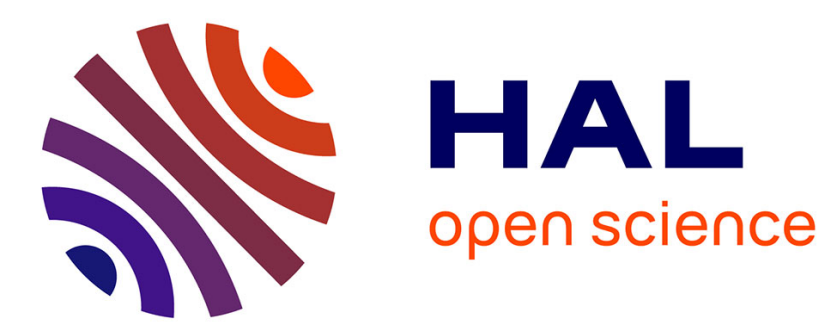

\title{
Etude des sauts atomiques dans les quasi-cristaux
}

\author{
S. Lyonnard, G. Coddens, B. Sepiol, R. Bellissent, Y. Calvayrac
}

\section{To cite this version:}

S. Lyonnard, G. Coddens, B. Sepiol, R. Bellissent, Y. Calvayrac. Etude des sauts atomiques dans les quasi-cristaux. Journal de Physique IV Proceedings, 1996, 06 (C2), pp.C2-71-C2-76. 10.1051/jp4:1996210 . jpa-00254188

\section{HAL Id: jpa-00254188 https://hal.science/jpa-00254188}

Submitted on 1 Jan 1996

HAL is a multi-disciplinary open access archive for the deposit and dissemination of scientific research documents, whether they are published or not. The documents may come from teaching and research institutions in France or abroad, or from public or private research centers.
L'archive ouverte pluridisciplinaire HAL, est destinée au dépôt et à la diffusion de documents scientifiques de niveau recherche, publiés ou non, émanant des établissements d'enseignement et de recherche français ou étrangers, des laboratoires publics ou privés. 


\title{
Etude des sauts atomiques dans les quasi-cristaux
}

\author{
S. Lyonnard, G. Coddens, B. Sepiol*, R. Bellissent and Y. Calvayrac** \\ Laboratoire L. Brillouin, CE Saclay, France \\ * IFF Universität Wien, Austria \\ ** CECM-CNRS, 15 rue G. Urbain, 94407 Vitry, France
}

\begin{abstract}
We present here new results on phason dynamics (atomic hopping) in quasi-crystals. An iron jump has been observed by spectroscopy Mössbauer in the system AlFeCu. Its relaxation time is very different from the one of the $3.9 \AA \mathrm{Cu}$ jump previously determined by neutron scattering. Two distinct atomic jump times have also been observed by neutron scattering in the system AlMnPd. They exhibit the same unusual temperature dependence as the $\mathrm{Cu}$ one, such that it seems to be universal.
\end{abstract}

\section{INTRODUCTION}

En 1984, Shechtman et al [1] découvrent des alliages métalliques dont les clichés de diffraction sont en apparence incompréhensibles. Il s'agit en effet de distributions ordonnées de pics de Bragg fins (caractéristiques des matériaux cristallins), mais possédant une symétrie d'ordre 5 incompatible avec la périodicité et l'ordre translationnel. Le paradoxe est cependant levé en considérant une notion d'ordre à longue distance plus générale que celle de périodicité: la quasi-périodicité. Les surprenants alliages sont alors baptisés quasi-cristaux. Ills sont non périodiques mais construits selon des règles d'assemblage strictes et donc très ordonnés à longue distance.

Les quasi-cristaux sont décrits par une méthode de coupe et projection [2] dans des espaces périodiques de dimensions supérieures. Cette projection introduit les lois complexes qui régissent l'ordre quasi-périodique. Considérons l'exemple de la construction d'un quasi-cristal à une dimension (Fig.1). Le super-espace est alors un réseau carré périodique bidimensionnel dont les noeuds sont décorés de batonnets appelés surfaces atomiques. L'espace physique, ou quasi-cristal monodimensionnel, est représenté par une droite de pente irrationnelle par rapport aux directions du réseau. Lorsqu'un batonnet coupe la droite, on définit une position atomique du QC : on obtient ainsi une suite quasi-périodique de segments longs $L$ et courts $C$ (suite de Fibonacci). Tout déplacement de la droite physique parallèlement à elle-même conduit à la construction d'un autre QC équivalent. Le choix d'une droite de pente rationnelle approximant la pente irrationnelle du QC définit une structure périodique dite approximante. (A). Les surfaces atomiques étant discontinues, le passage du QC à l'approximant se fait par l'intermédiaire de sauts atomiques appelés phasons (Fig.2). Les phasons échangent une séquence LC en une séquence CL.

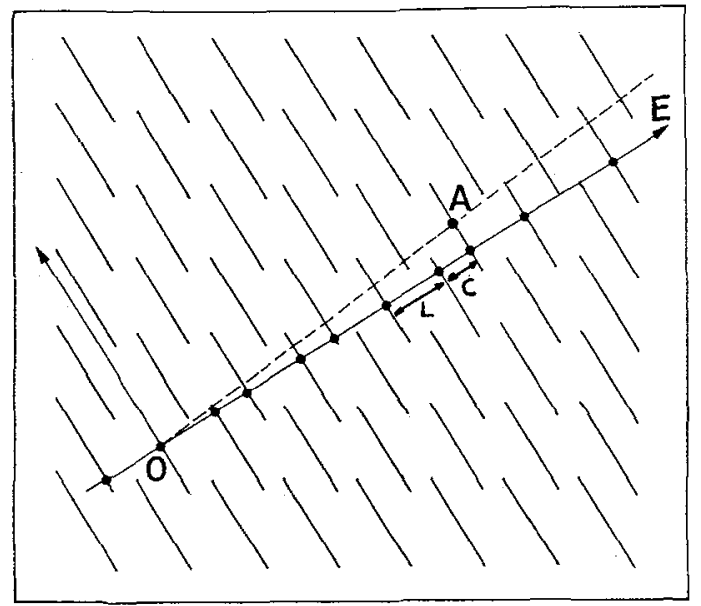

Fig 1 : Méthode de coupe et projection

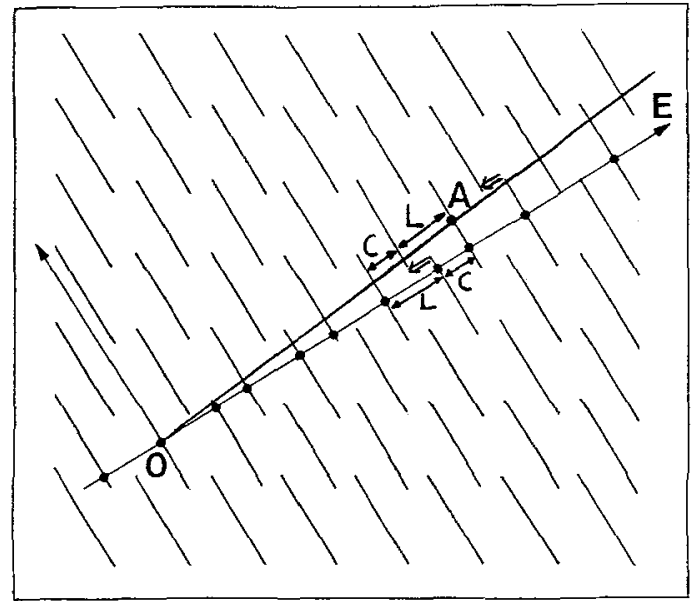

Fig 2 : Mise en évidence des phasons (=) 
Les quasi-cristaux bidimensionnels s'obtiennent par projections d'espaces de dimension au moins 4. Ils peuvent être également représentés par des pavages quasi-périodiques de type Penrose (Fig.3), construits à partir de deux cellules initiales. Les phasons sont alors décrits comme des flip-flop ou réarrangements locaux de ces deux tuiles élémentaires. Ils correspondent à des sauts atomiques entre positions voisines énergétiquement équivalentes (changement de perspective dans les "cubes" qui apparaissent dans le pavage).
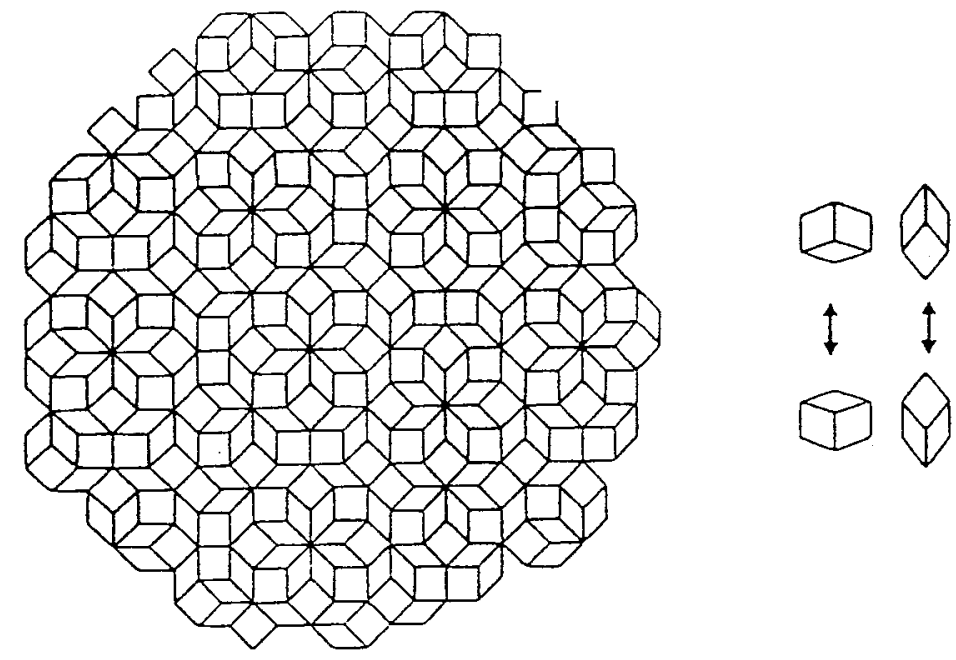

Fig 3 : Pavage de Penrose octogonal. Deux possibilités de flip-flop sont illustrées à droite.

Nos travaux ont montré que les phasons existent à haute température dans les quasi-cristaux. L'étude de la dynamique de ces sauts est importante pour la compréhension d'un certain nombre de mécanismes physiques. La connaissance des espèces atomiques impliquées dans les sauts et des distances de saut peut permettre en particulier de tester les modèles structurels (Katz et Gratias [3]). D'autre part, les mécanismes de diffusion (proposés par Kalugin et Katz [4]) et de transitions de phases QCapproximants pourraient être initiés par les phasons. Enfin, les phasons interviennent dans le scénario de stabilisation par entropie des quasi-cristaux, et constituent donc un processus fondamental dans le modèle du Random Tiling [5].

Une expérience de diffusion quasi-élastique de neutrons menée à Saclay sur le spectromètre de temps de vol Mibémol a été précédemment réalisée sur un échantillon d'AlFeCu [6]. Elle a mis en évidence l'existence d'une Lorentzienne quasi-élastique au pied du pic élastique, interprétée comme la signature du processus de saut. Des substitutions isotopiques successives ont démontré que le signal est entièrement proportionnel à la section efficace de diffusion du Cuivre : le saut observé est donc celui du Cuivre. Une dépendance inattendue en température et en vecteur de diffusion a été observée. Une distance de saut de $3.9 \AA$ A, un temps de relaxation de $12 \mathrm{ps}$, et une énergie d'activation de $750 \mathrm{meV}$ ont été déterminés.

De plus, une expérience de spectroscopie Mössbauer dans le même système a permis de mettre en évidence les sauts du Fer. Les temps de relaxation des deux types d'atomes sont très différents. Enfin, les phasons ont été récemment observés dans un alliage quasi-cristallin d'AIMnPd par diffusion des neutrons. Ces nouveaux résultats sont analysés par la suite.

\section{LE SAUT DU FER DANS AIFeCU}

L'expérience de spectroscopie Mössbauer a été réalisée à Vienne (Autriche) sur un échantillon de poudre icosaédrique parfaitement monophàsée de composition $\mathrm{Al}_{62} \mathrm{Fe}_{12.5} \mathrm{Cu}_{25.5}$. Le dispositif expérimental se compose d'une source de ${ }^{57} \mathrm{Co}$ émettant des rayons $\gamma$ d'énergie $14.4 \mathrm{keV}$, d'un dispositif laser permettant la calibration de la vitesse de translation de la source, d'un porte-échantillon monté dans un four et d'une chambre d'ionisation au $\mathrm{Kr}$ (détecteur). L'épaisseur de l'échantillon est optimisée pour limiter l'absorption due à des processus compétitifs (diffusion Compton, diffusion Rayleigh...).

De façon à visualiser un signal quasi-élastique large dans la zone hors-résonance, des conditions opératoires particulières doivent être choisies : 
- Le rapport signal sur bruit est mauvais dans une expérience de transmission, particulièrement dans la zone hors résonance où l'intensité comptée est maximale et les fluctuations statistiques importantes. Il est donc très difficile à priori de détecter un signal quasi-élastique de faible intensité. On a en conséquence utilisé un échantillon enrichi à $95 \%$ en ${ }^{57} \mathrm{Fe}$ pour améliorer le contraste (gain d'un facteur 2000).

- La deuxième difficulté de l'expérience réside dans la trop bonne résolution du dispositif (de l'ordre d'une dizaine de neV). Pour pouvoir détecter un signal très large de quelques $\mu \mathrm{eV}$, il est nécéssaire d'explorer la plus grande zone hors résonance possible. Ceci impose le choix de grandes vitesses de translation de la source $(160 \mathrm{~mm} / \mathrm{s})$, et donc l'utilisation d'un dispositif de calibration particulier. En effet, la calibration des petites vitesses (de l'ordre de quelques $\mathrm{mm} / \mathrm{s}$ ) se fait habituellement par la mesure du splitting hyperfin du Fer dans la source. Dans notre cas, les effets fins (magnétiques ou électriques) ne sont plus visibles et le pic de résonance est distordu par l'effet d'une très forte absorption. Il est donc impossible d'utiliser les méthodes traditionnelles. Un système optique interféromètrique est par conséquent mis en place.

Dans de telles conditions, un signal quasi-élastique indétectable à basse température $\left(660^{\circ} \mathrm{C}\right)$ est mis en évidence à haute température $\left(780^{\circ} \mathrm{C}\right)$. La figure 4 montre pour ces deux températures les résidus obtenus avec un ajustement des spectres à deux composantes Lorentziennes (pour tenir compte du splitting du Fer). L'ajustement n'est pas adapté à $780^{\circ} \mathrm{C}$ : une déviation systématique des points expérimentaux indique la présence d'une troisième composante, interprétée comme la signature des sauts du Fer dans l'échantillon. L'ajustement réalisé avec trois composantes indique une largeur quasi-élastique de $4 \mu \mathrm{eV}$ correspondant à un temps de relaxation de $164 \mathrm{ps}$.

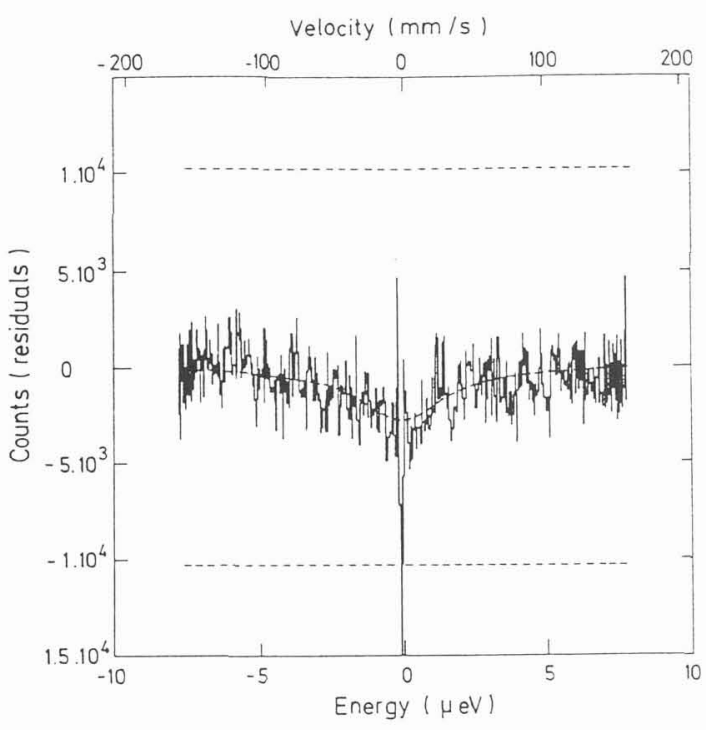

a)

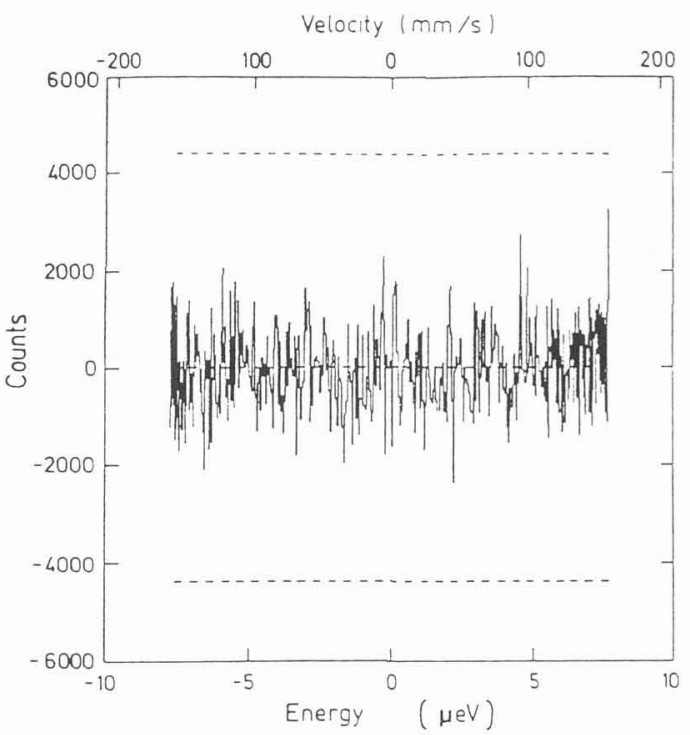

b)

Fig. 4 : (a) résidus à $780^{\circ} \mathrm{C}$

(b) résidus à $660^{\circ} \mathrm{C}$.

Les lignes pointillées indiquent $0.5 \%$ de l'intensité élastique.

Deux sauts d'espèces atomiques différentes ont ainsi été observés dans AlFeCu. Ils se produisent suivant des échelles de temps très différentes : $164 \mathrm{ps}$ pour le Fer et $12 \mathrm{ps}$ pour le Cuivre. Une telle observation confirme donc l'interprétation des résultats en termes de sauts atomiques et écarte toute hypothèse fondée sur des vibrations de clusters ou des mouvements collectifs. 


\section{ETUDE DES PHASONS DANS AMMPA PAR DIFFUSION DE NEUTRONS}

Une expérience de diffusion quasi-élastique des neutrons a été faite sur le spectromètre de temps de vol Mibémol au Laboratoire Léon Brillouin à Saclay. L'échantillon utilisé est une poudre de quasi-cristal parfait non magnétique de composition $\mathrm{Al}_{70.3} \mathrm{Mn}_{21.4} \mathrm{Pd}$ 8.3. Il est placé dans un cylindre de Saphir à l'intérieur d'un container de Niobium et monté dans un four sous vide. Les détecteurs d' ${ }^{3} \mathrm{He}$ sont positionnés en plusieurs groupes d'angle de diffusion 20 . Deux séries de mesures ont été faites à de grandes longueurs d'ondes incidentes (compatibles avec une bonne résolution) pour explorer le plus grand domaine possible en vecteur de diffusion. Deux temps de relaxation différents ont été déterminés. Une étude en température et en $\mathbf{Q}$ a été menée pour chacun d'entre eux.

Dans chaque cas, les données ont été complétées par un spectre de boite vide à haute et basse températures pour la correction du bruit de fond, et par un spectre de Vanadium à température ambiante pour l'efficacité des détecteurs et la fonction de résolution de l'appareil $R(Q, \omega)$. Les spectres corrigés et normalisés ont été ajustés à l'aide de trois composantes convoluées à $R(Q, \omega)$ :

$$
\left[A_{0}(Q) \delta(\omega)+A_{1}(Q) \frac{1}{\pi} \frac{\Gamma}{\omega^{2}+\Gamma^{2}}+A_{2}(Q, \omega)\right] \otimes R(Q, \omega)
$$

La mesure Dirac correspond au pic de diffusion élastique d'intensité $A_{0}(Q)$. $A_{2}(Q, \omega)$ est un terme de Debye utilisé pour décrire la densité d'état de phonons de basse énergie (une dépendance linéaire en temps de vol ne convient pas dans la mesure où la fenêtre utilisée en $Q$ est large). La composante Lorentzienne d'intensité $A_{1}(Q)$ et de largeur $\Gamma$, située au pied du pic élastique, est interprétée comme la signature des sauts de temps de relaxation $\tau=\hbar / \Gamma$. L'intensité $A_{1}(Q)$ est de l'ordre de $1 \%$ de $A_{0}(Q):$ le signal est donc très difficile à observer et les ajustements doivent être faits en excluant le pic élastique.

\subsection{Dépendance en température}

Les deux séries de mesures ont été effectuées dans la même gamme de température : de la température ambiante à $800^{\circ} \mathrm{C}$ environ. La dépendance observée est du mếme type dans les deux cas. Les sauts sont activés thermiquement : l'intensité quasi-élastique est maximale à la plus haute température et décroit continument jusqu'à $500^{\circ} \mathrm{C}$, où elle devient indétectable (Fig 6).

Dans un modèle simple de sauts d'une particule entre deux sites équivalents (double puits de potentiel), on s'attend à ce que l'intensité du signal reste constante avec la température et à ce que la largeur suive une loi d'Arrhénius de la forme $\hbar / \Gamma=\tau=\tau_{0} \exp \left(-\mathrm{E}_{\mathrm{a}} / \mathrm{kT}\right)$, où $\mathrm{E}_{\mathrm{a}}$ est l'énergie d'actication du processus de saut. Or on observe expérimentalement le comportement inverse : la largeur semble constante, dans la limite de précision des mesures, et l'intensité varie suivant une loi d'Arrhenius (Fig.5). On détermine ainsi deux énergies d'activation : $\mathrm{E}_{1}=271 \mathrm{meV}$ et $\mathrm{E}_{2}=177 \mathrm{meV}$, auxquelles correspondent deux temps de relaxation différents.

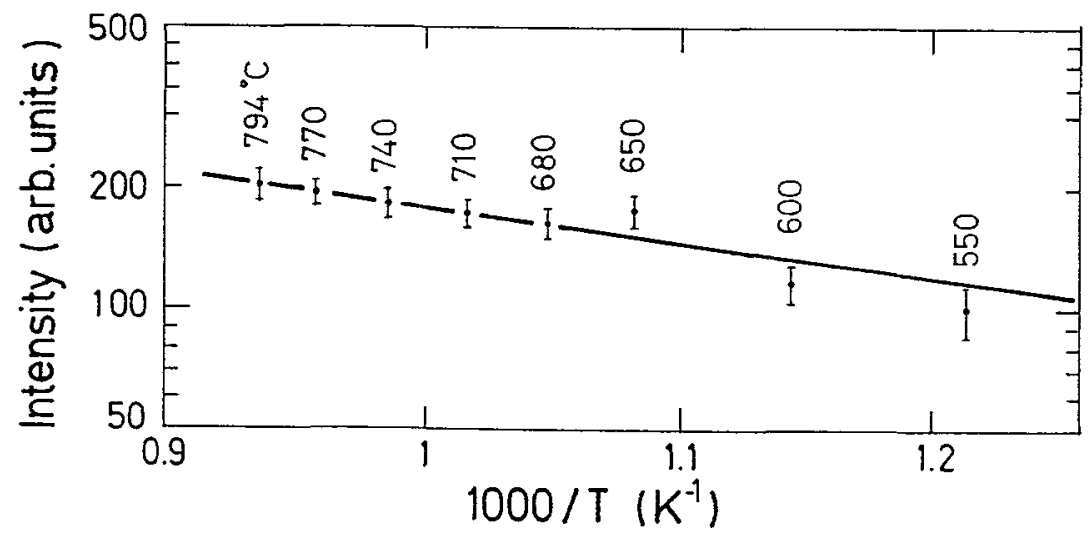

Fig 5 : Variation de 1'intensité du signal quasi-élastique en fonction de la température. 
Ce comportement inhabituel semble caractéristique d'un mécanisme assisté. Dans le cas précédent du Cuivre dans AlFeCu, l'énergie d'activation de $750 \mathrm{meV}$ était de l'ordre de grandeur de l'énergie de création d'une lacune d'Al. On pouvait donc envisager une assistance par diffusion de lacunes. Pour AlMnPd, les énergies trouvées sont davantage compatibles avec une assistance par phonons. Quoiqu'il en soit, l'observation d'une même dépendance en température dans deux alliages différents semble indiquer qu'il s'agit d'un comportement systématique dans les quasi-cristaux.
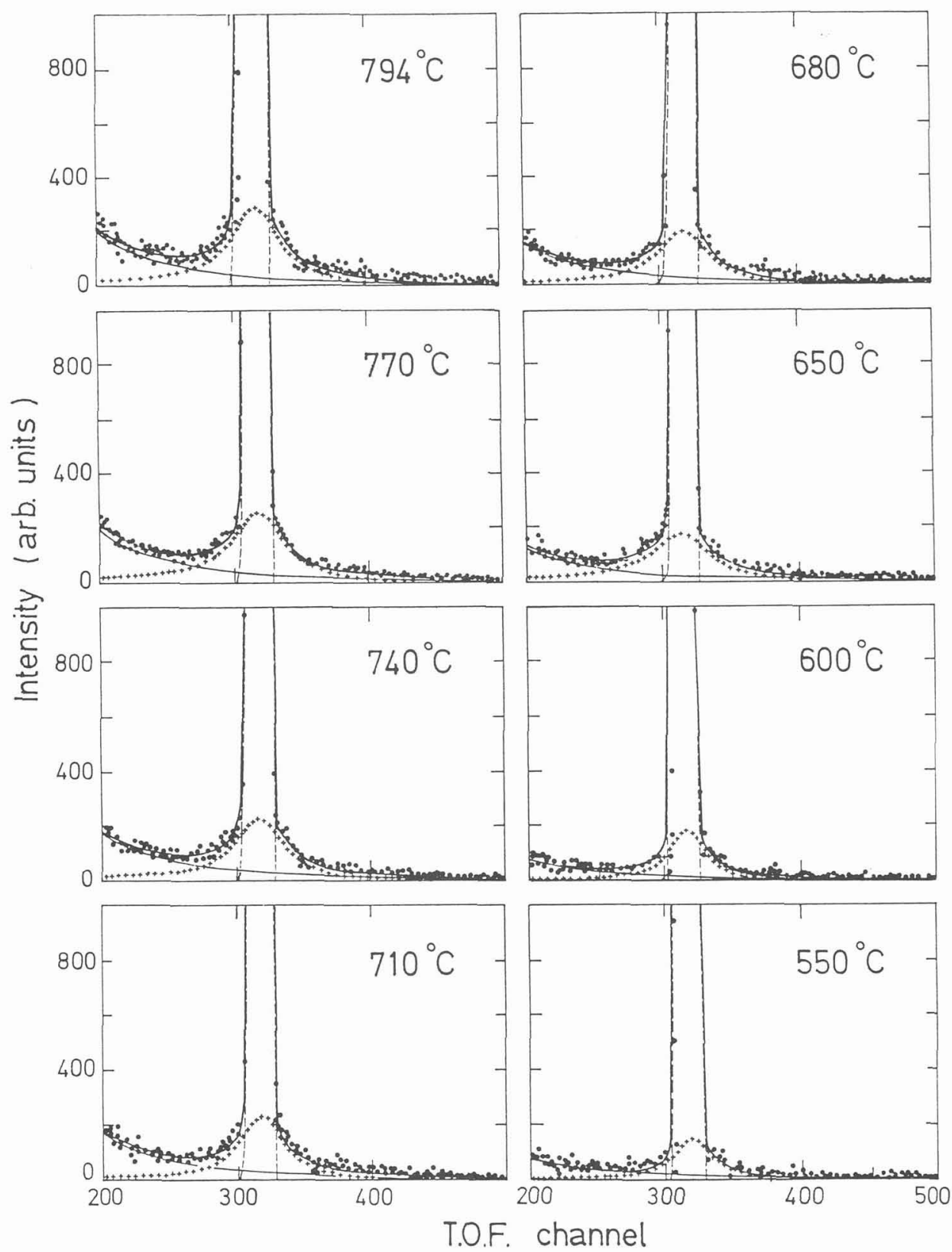

Fig.6 : Evolution du signal en fonction de la température. 


\subsection{Dépendance en vecteur de diffusion $Q$}

La dépendance en $Q$ est extrêmement délicate à déterminer. En effet, seuls les 5 derniers groupes d'angles donnent des intensités suffisamment élevées pour permettre l'ajustement des données. De plus, les spectres sont pollués aux faibles valeurs de $Q$ par des parasites (phonons, réflexions de Bragg...). Seule une évolution qualitative du signal peut être décrite. Pour chaque série de mesure, la largeur de la Lorentzienne correspondante reste constante tandis que l'intensité croit avec Q. Dans le modèle de saut entre deux sites distants de d, le facteur de forme quasi-élastique est une fonction de Bessel (1-jo(Qd)), qui atteint son premier maximum en $\mathrm{d}$.

Cependant, la fenêtre en $Q$ de l'expérience n'est pas ici suffisamment étendue pour permettre l'observation d'un maximum. Il est d'autre part impossible d'utiliser de plus petites longueurs d'onde sans dégrader considérablement la résolution du spectromètre (l'expérience a déjà été réalisée dans les conditions les plus limites). Aucune distance de saut ne peut donc être déterminée par cette technique.

\section{CONCLUSIONS}

Plusieurs expériences successives utilisant des techniques complémentaires, et réalisées sur des échantillons quasi-cristallins différents, ont démontré l'existence d'un signal quasi-élastique activé thermiquement. Ce signal correspond à des sauts atomiques locaux extrêmement rapides et assistés par des processus encore inconnus (diffusion de lacunes, phonons, breathers...). Tous les atomes sont impliqués dans le mécanisme de saut, suivant des échelles de temps et des distances variables. Nos résultats n'indiquent pas l'existence d'une diffusion rapide, comme celle prévue par Kalugin et Katz: les sauts sont au contraire confinés dans l'espace. Il est parfois nécéssaire que plusieurs sauts se produisent avant qu'un saut particulier soit rendu possible. Dans ces conditions, les flip-flop décrits dans les pavages de Penrose ne peuvent pas se propager facilement à longue distance.

\section{Références :}

[1] D.Shechtman, I.Blech, D.Gratias, J.W.Cahn, Phys. Rev. Lett. 53 (1984) 1951

[2] M.Duneau, A.Katz, Phys. Rev. Lett.. 47 (1986) 181.

[3] A.Katz, D.Gratias, J. Non-Cryst. Solids 153-154 (1993) 187

[4] P.A.Kalugin, A.Katz, Europh. Lett. 21 (1993) 921

[5] M.Widom, D.P.Deng, C.L.Henley, Phys. Rev. Lett. 63 (1989) 310; K.Strandburg, L.-H. Tang, M.V.Jaric, Phys. Rev. Lett. 63 (1989) 314.

[6] G.Coddens, C.Soustelle, R.Bellissent, Y.Calvayrac, Europhys. Lett. 23 (1993) 33; G.Coddens, Ann. Chim. France 18 (1993) 513. 